\title{
Integrated Cytogenetic and High-Resolution Array CGH Analysis of Genomic Alterations Associated with MYCN Amplification
}

\author{
A. Pandita ${ }^{a} \quad$ J. Bayani ${ }^{b} \quad$ J.Paderovac P. Marranoc $\quad$ C. Graham ${ }^{b} \quad$ M. Barrett ${ }^{d}$ \\ M. Prasad ${ }^{e} \quad$ M. Zielenska ${ }^{f} \quad$ J.A. Squire ${ }^{g}$ \\ ${ }^{a}$ Department of Oncology Diagnostics, Genentech, South San Francisco, Calif., USA; ${ }^{b}$ Department of \\ Laboratory Medicine and Pathobiology, University of Toronto, and ${ }^{\mathrm{C}}$ Department of Applied Molecular Oncology,

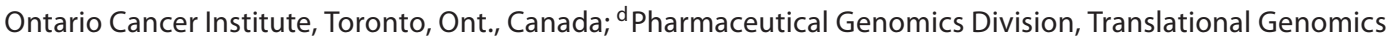 \\ Research Institute, Scottsdale, Ariz., ${ }^{e}$ Agilent Technologies Inc. Headquarters, Santa Clara, Calif., USA; \\ fDepartment of Pathology and Laboratory Medicine, The Hospital for Sick Children, Toronto, Ont., and \\ gDepartment of Pathology and Molecular Medicine, Queen's University and Kingston General Hospital, \\ Kingston, Ont., Canada
}

\section{Key Words}

$\mathrm{aCGH} \cdot \mathrm{FISH} \cdot \mathrm{mBAND} \cdot \mathrm{MYCN} \cdot \mathrm{Neuroblastoma} \cdot$

Retinoblastoma

\begin{abstract}
Amplification of oncogenes and closely linked flanking genes is common in some types of cancer and can be associated with complex chromosome rearrangements and/or co-amplification of non-syntenic chromosomal regions. To better understand the etiology and structural complexity of focal MYCN amplicons in human neuronal cancer, we investigated the precise chromosomal locations of high copy number genomic regions in MYCN amplified cell lines. An integrated cytogenetic map of the MYCN amplicon was created using high-resolution array $\mathrm{CGH}$, spectral karyotyping (SKY), multi-color banding (mBAND), and fluorescence in situ hybridization (FISH) in 4 human neuronal tumor cell lines. The evidence of complex intra- and inter-chromosomal events, providing clues concerning the nature of the genomic mechanisms that contributed to the process of MYCN amplification, was observed. The presence of multiple co-amplified syntenic or non-syntenic sequences in the MYCN am-
\end{abstract}

\section{KARGER}

Fax +4161306 1234

E-Mail karger@karger.ch

www.karger.com
C) 2011 S. Karger AG, Basel

$1424-8581 / 11 / 1341-0027 \$ 38.00 / 0$

Accessible online at:

www.karger.com/cgr plicon is quite intriguing. MYCN is usually centrally located in the amplicon; however, the structure and complexity of the amplicons were highly variable. It is noteworthy that clusters of unstable repetitive regions characterized by CNV sequences were present throughout the regions encompassed by MYCN gene amplification, and these sequences could provide a mechanism to destabilize this region of the genome. Complex structural rearrangements involving genomic losses and gains in the $2 \mathrm{p} 24$ region lead to MYCN amplification and that these rearrangements can trigger amplification events.

Copyright $\odot 2011$ S. Karger AG, Basel

Gene amplification is a major mechanism of oncogene activation, which in many cases directly contributes to the capacity of cancer cells to grow autonomously [Albertson et al., 2003]. High copy number MYCN amplification has been observed in neuroblastomas [Brodeur et al., 1984; Schwab and Amler, 1990] and retinoblastomas

\section{A. Pandita and J. Bayani contributed equally to this work.}

Jeremy A. Squire, $\mathrm{PhD}$

Richardson Laboratories, Room 201e

88 Stuart St, Queen's University

Kingston, ON K7L 3N6 (Canada)

Tel. +1 613533 2345, E-Mail squirej@ queensu.ca 
[Squire et al., 1986] and is associated with poor prognosis of neuroblastomas. In primary neuronal tumors, MYCN amplicons often exist as autonomously replicating extrachromosomal elements called double minutes (dmins) [Moreau et al., 2006], while in neuronal cell lines, MYCN amplicons are usually integrated into the chromosome and appear as homogeneously staining regions (hsrs) [Kitajima et al., 2001; Shimizu et al., 2001]. It has been proposed that $M Y C N$-containing hsrs may arise during in vitro cell growth by a process involving the reintegration of dmins at a site that underwent amplification by unequal sister-chromatid exchange [Narayanan et al., 2006]; such chromosomal breaks and/or excision of genomic sequences play roles in amplicon formation in cancer cells. Neither the complexity of MYCN amplicons nor the mechanisms by which they form in primary tumors and tumor-derived cell lines are well characterized.

Most MYCN amplicons contain 50-100 tandem repeats of a unit that is $100 \mathrm{~kb}$ to $1 \mathrm{MB}$ in size [Akiyama et al., 1993; Noguchi et al., 1996]. MYCN maps to the central core of amplicons and co-amplified genomic regions vary considerably in size and complexity [Kohl et al., 1983; Schwab et al., 1983, 1984; Pandita et al., 1997]. Studies have shown that despite the high levels of complexity between different cell lines, the high-expression level of MYCN is considered to be driven by DNA copy-number increases, with the most amplified cell lines expressing the highest levels of the gene [reviewed in Schwab, 2000]. Array comparative genomic hybridization (aCGH) has recently been used to precisely characterize the structure and location of high copy number focal MYCN amplicons in cancer cells [Mosse et al., 2007; Caren et al., 2008; Fix et al., 2008]. These studies revealed single continuous focal amplicons centered on the gene at 2p24, with or without multiple discrete co-amplifications in $2 \mathrm{p}$ and/or additional non-syntenic chromosome regions [Mosse et al., 2007; Caren et al., 2008; Fix et al., 2008]. However, these aCGH studies did not provide sufficient data to determine the cytogenetic relationships between genomic sequences co-amplified with MYCN or to deduce putative mechanisms by which these amplicons formed.

The goal of this study was to apply aCGH and advanced molecular cytogenetic methods to better understand the etiology and structural complexity of focal MYCN amplicons in human neuronal cancer. Because each molecular cytogenetic technique possesses its own set of limitations [Bayani and Squire, 2007], aCGH, spectral karyotyping (SKY), multi-color banding (mBAND) and fluorescence in situ hybridization (FISH) analyses were used to better determine the precise chromosomal locations of all high copy number genomic regions in 4 human neuronal tumor cell lines (Y79, GOTO, LAN5 and IMR32). High copy number focal amplification of multiple chromosome regions were observed in Y79, GOTO and IMR32 cell lines. These amplifications were associated with structural rearrangements proximal to $M Y C N$ and deletions within and distal to 2p24. LAN5 carried one dmin population with focal amplification centered at $M Y C N$ and a second dmin population with a focal amplicon from $6 \mathrm{p} 12$. Interestingly, in all 4 cell lines, unstable repetitive regions characterized by copy-number variation (CNV sequences) occurred in close proximity to the copy-number transition zones of high copy number amplicons. These data suggest that complex structural rearrangements involving genomic losses and gains in the 2p24 region lead to MYCN amplification in neuronal cancer cells, and that these rearrangements can be accompanied by intra- or interchromosomal alterations which facilitate high copy number focal amplifications of MYCN.

\section{Materials and Methods}

\section{Cell Lines}

The neuroblastoma cell lines LAN5, GOTO, IMR32, and retinoblastoma cell line Y79 were maintained as previously described by Pandita et al. [1997] and processed for cytogenetic harvest [Bayani and Squire, 2004c]. DNA was extracted by standard methods [Bayani and Squire, 2004b]. Normal human lymphocytes were also prepared for cytogenetic harvest.

\section{Array Comparative Genomic Hybridization}

DNAs were extracted from each cell line using standard extraction methods. aCGH was performed using the Agilent $44 \mathrm{~K}$ and $244 \mathrm{~K}$ platform (Agilent Technologies, Santa Clara, Calif., USA), according to the manufacturer's instructions and as described by Prasad et al [2008]. Slides were dried and further scanned using an Agilent 2565AA DNA microarray scanner. Microarray images were analyzed using Agilent Feature Extraction software version 8.1. Default settings for CGH arrays were used according to the supplier's recommendations. CGH Analytics software version 3.4 (Agilent Technologies) was used for analysis. The data quality of each microarray was assessed using the Quality Metrics report generated by the Agilent CGH analytics software (v.3.4). This report provides acceptable ranges for qualitycontrol parameters from each microarray experiment, based on analysis of numerous well-characterized cell lines. All CGH data represent the average of dye-flip experiments for each of the samples, using the aberration detection method 1 (ADM-1), which is based on computing significance ratios for all genomic intervals [Lipson et al., 2006]. To make aberration calls, a robust estimate of the noise was obtained by calculating the spread of the ratio differences between consecutive probes (DLRSpread) along all chromosomes. The ability of the DLRSpread to measure the noise of the ratios independently of the number and severity of aberrations was used to assess the overall quality of each experiment. 
The ADM-1 algorithm searches for chromosomal intervals of any length for which the mean interval ratio is significantly different from zero. An interval has a ratio significantly different from zero if it is more than the threshold standard deviations. For this study, the threshold was optimized to a value of 10 to reduce inherent sample noise, without negating true consistent imbalances along each chromosome. Further details concerning these principles are available at the Agilent website (www.agilent.com/chem/ goCGH). Aberrations were assigned as either gain (ratio $\geq 0.5$ ), loss $(\leq-1.0)$ or amplification $(\geq 2.0)$. Copy number variation (CNV) loci were identified using the Agilent supplied annotated files. Source files for array experiments are available in the supplementary material (for all online supplementary material see www.karger.com/doi/10.1159/000324698).

\section{Molecular Cytogenetics}

Cytogenetic preparations from each cell line and normal controls were aged at room temperature for at least 1 week prior to use. Bacterial artificial chromosome (BAC) cultures were procured from the Applied Centre for Genomics, Toronto, Ont., Canada (http://www.tcag.ca/) and selected according to the UCSC Genome Bioinfomatics Browser (http://genome.ucsc.edu/, March 2006 Build). The BACs used for this study are listed in online supplement table 1. DNA from the BAC cultures were extracted by standard protocols [Bayani and Squire, 2004c] and directly labeled with either Spectrum Green-dUPT (Abbott Molecular), Spectrum Orange-dUPT (Abbott Molecular), Cy5.5 (Applied Biosystems Inc.), or diethylaminocoumarin (DEAC)-dUPT (Applied Biosystems Inc.), using the Vysis Nick Translation Kit (Abbott Molecular). The labeled probes were then precipitated in the presence of excess Human Cot-1 DNA (Invitrogen, Canada) and sonnicated salmon sperm DNA (Sigma, Canada) and resuspended in Hybrisol VII (Qbiogene). The slides were pepsin treated, dehydrated and co-denatured with the probe for $2 \mathrm{~min}$ at $75^{\circ} \mathrm{C}$ using the Hybrite System (Abbott Molecular) and allowed to hybridize overnight at $37^{\circ} \mathrm{C}$. The following day, the slides were processed using a Rapid Wash protocol consisting of one 3 -min wash at $75^{\circ} \mathrm{C}$ in $0.3 \% \mathrm{NP}-$ $40 / 0.1 \times$ SSC, and a $10-\mathrm{min}$ wash at room temperature in $0.1 \% \mathrm{NP}-$ $40 / 2 \times$ SSC. The slides were briefly rinsed in $1 \times$ SSC and mounted in DAPI/Antifade (Vectashield). Each labeled BAC was initially tested on normal human lymphocyte metaphases to ensure that the BAC mapped to the correct chromosomal location. The slides were visualized using the Metasystems Imaging System (Altlussheim, Germany) equipped with an AxioPlan Fluorescent Microscope (Carl Zeiss, Canada). A centromere 2 probe and a whole chromosome 18 paint were purchased from Abbott Molecular and used according to the manufacturer's instructions.

Cytogenetic specimens were processed as described above. The mBAND painting probe for chromosome 2 was purchased from Metasystems (Metasystems, Altlussheim, Germany) and the procedure carried out according to the manufacturer's instructions. Image analysis and capture was performed using the Metasystems Imaging System (Metasystems) equipped with an AxioPlan Fluorescent Microscope (Carl Zeiss, Canada), as described previously by Lim et al. [2004] and Paderova et al. [2007].

Cytogenetic specimens were processed as described above and hybridized with the SKY-painting probes (Applied Spectral Imaging, Vista, Calif., USA) as per the manufacturer's instructions. Image analysis and capture was performed using an AxioPlan Fluorescent Microscope and Spectral Karyotyping software (Applied Spectral Imaging).

Integrative Genomics of the $M Y C N$ Amplicon

\section{Results}

The following experiments were designed to identify and fine-map MYCN amplicons and all associated high copy-number variations in Y79, GOTO, IMR32, and LAN5. For this purpose, SKY, mBAND, aCGH, and FISH analyses were conducted for each cell line (see Materials and Methods and legends to figures for detailed experimental procedures). Supplemental tables summarize the BAC clones used in this study (online suppl. table 1), integrated SKY/mBAND karyotypes (online suppl. table 2) for each cell line and genes mapping to amplified regions in GOTO (online suppl. table 3) and Y79 (online suppl. table 4). Figures 1, 3, 5 and 7 show representative SKY and mBAND data and a graphic summary of chromosome 2 aCGH data for Y79, GOTO, IMR32, and LAN5, respectively. Figures 2, 4, 6 and 8 show representative locusFISH analyses of Y79, GOTO, IMR32, and LAN5, respectively. Complete aCGH files and SKY karotypes are also available as supplementary files and figures.

\section{Integrated Molecular Cytogenetic and Genomic Analysis of Y79: Complex Interchromosomal Rearrangements Associated with MYCN Amplification}

SKY analysis of Y79 showed a nearly diploid karyotype with relatively simple structural aberrations (online suppl. table 2; online suppl. fig. 1). Y79 has an hsr on a der(1) chromosome involved in a complex structural rearrangement with chromosomes 2 and 18 (fig. 1A, bottom), that includes portions of chromosome 2 , from $2 \mathrm{p} 12 \rightarrow 2 \mathrm{p} 16$ and the 2p24 locus containing MYCN. This hsr includes 50 copies of MYCN [Godbout and Squire, 1993; Pandita et al., 1997] as well as amplified DNA sequences derived from chromosome 18 [Godbout et al., 1997]. Chromosome 2 -specific mBAND analysis confirmed the presence of the $2 \mathrm{p} 12 \rightarrow 2 \mathrm{p} 16$ region and amplified sequences from 2p24 (fig. 1B, bottom) within the hsr that includes material from chromosome 18. The inability of SKY to detect the discrete chromosome 2 material within the regions of chromosome 18 comes from the similarity of the fluorophore combinations that make up these 2 probes within the SKY probe cocktail [Bayani and Squire, 2004a], making the integration of the aCGH data invaluable. Furthermore, aCGH, SKY and mBAND data confirmed losses of the terminal portion of $2 \mathrm{p}$ from $2 \mathrm{p} 25.2$ to 2 pter and a regional gain from 2 p22.1 to 2p11 (fig. 1C). The correspondence between aCGH, SKY and mBAND data for these aberrations is indicated graphically by the orange lines in figures $1 \mathrm{~B}$ and C. A net gain of one copy from $2 \mathrm{q} 24 \rightarrow 2 \mathrm{q} 36.3$

Cytogenet Genome Res 2011;134:27-39 


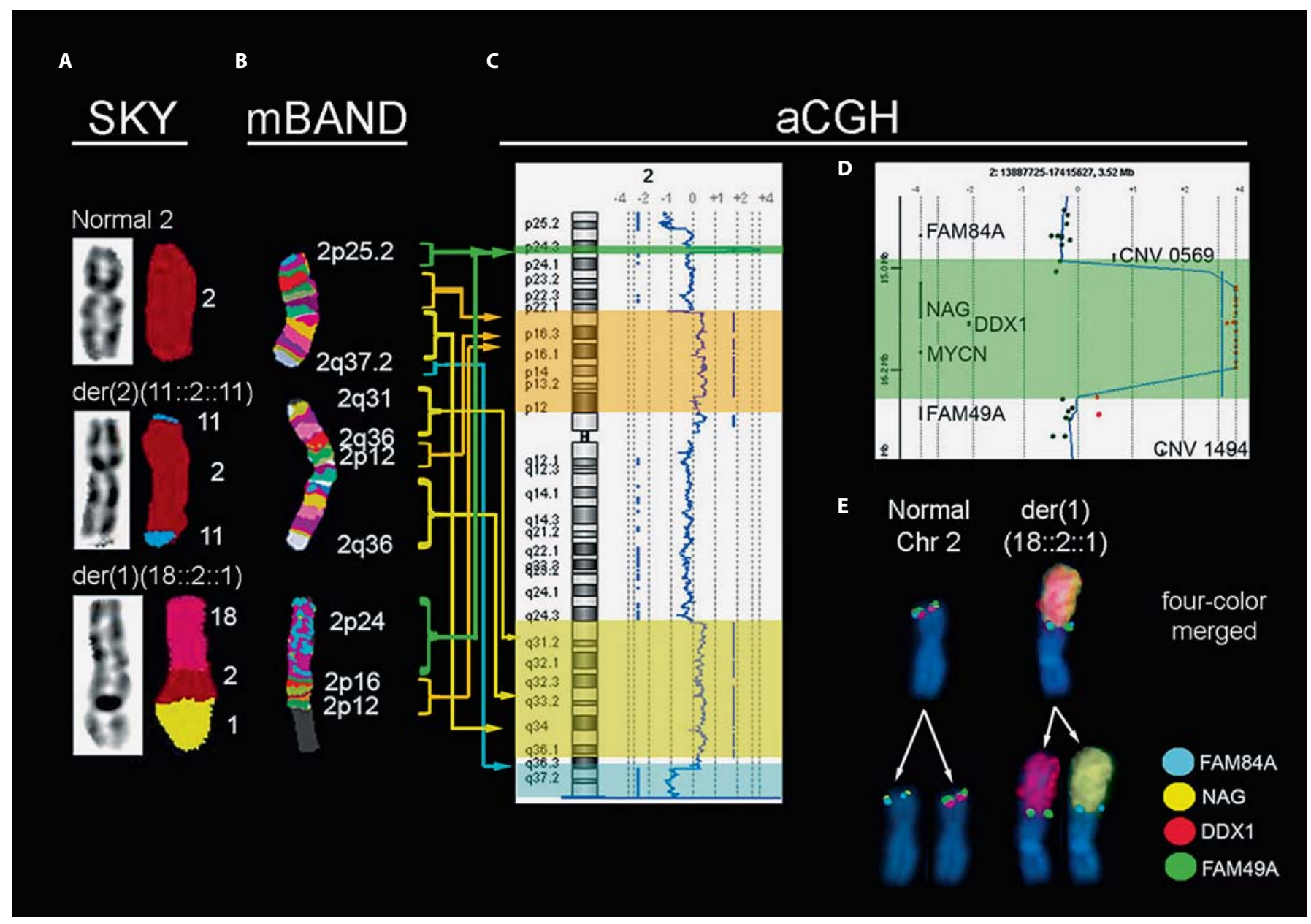

Fig. 1. Integrated molecular cytogenetic and genomic analyses of Y79. A SKY analysis shows one normal copy of chromosome 2 (top), a der(2) chromosome with sequences from chromosomes 2 and 11 (middle) and a complex der(1) chromosome with sequences from chromosomes 1, 2 and 18 (bottom). B Chromosome 2-specific mBAND analysis of these 3 chromosomes revealed the following: Top: a structurally intact chromosome 2; middle: a der(2) chromosome with a duplication of $2 \mathrm{q} 31 \rightarrow 2 \mathrm{q} 36$ joined at $2 \mathrm{p} 12$ and a terminal deletion of $2 \mathrm{q} 37$; and bottom: sequences derived from $2 \mathrm{p} 24$ and from $2 \mathrm{p} 16 \rightarrow \mathrm{p} 12$ on $\operatorname{der}(1)(18:: 2:: 1)$. C aCGH analysis of chromosome 2 shows amplification of 2 p24 centered around MYCN. Correspondence between aCGH gains

(yellow lines) and a terminal deletion of $2 \mathrm{q} 27$ were also confirmed by aCGH and mBAND data for Y79 (fig. 1B, $\mathrm{C})$. The copy-number transition point for the terminal deletion of 2p25 was marked with the presence of CNV1493. Discrete focal high-copy amplification of $M Y C N$ was observed at 2p24. Four-color FISH using BACs for FAM84A, NAG, DDX1, and FAM49A confirmed the co-amplification of NAG and DDX1 in the hsr along with MYCN; in and losses and copy-number changes detected by SKY and MBAND are shown by green, orange, yellow and aqua arrows between $\mathbf{B}$ and C. D High-resolution aCGH karyogram of the MYCN region in Y79. The amplicon includes NAG, DDX1 and MYCN, with CNV0569 in the telomeric copy-number transition region. E Four-color locusspecific FISH using probes for FAM84A, NAG, DDX1 and FAM49A showing co-amplification of DDX1 and NAG in the hsr and translocation of FAM49A and FAM84A on $\operatorname{der}(1)$ (18::2::1). Shown are the merged four-color images of the normal chromosome 2 and $\operatorname{der}(2)$ on the top, and comparable 2-color unmerged images of the same chromosome. The amplified region is mostly telomeric to FAM84A. Normal chromosome 2 is shown for comparison. contrast, CNV regions containing FAM49A and FAM $84 A$ were detected adjacent to but not in the amplicon at 2p24 (fig. 1D, right). The MYCN/DDX1/NAG amplicon lies proximal to FAM84A. At the resolution afforded by metaphase FISH, it was not possible to determine whether $M Y C N, D D X 1$ and $N A G$ are retained as single-copy loci in their usual genomic locations between FAM49A and FAM84A (fig. 1E) on der(1). 


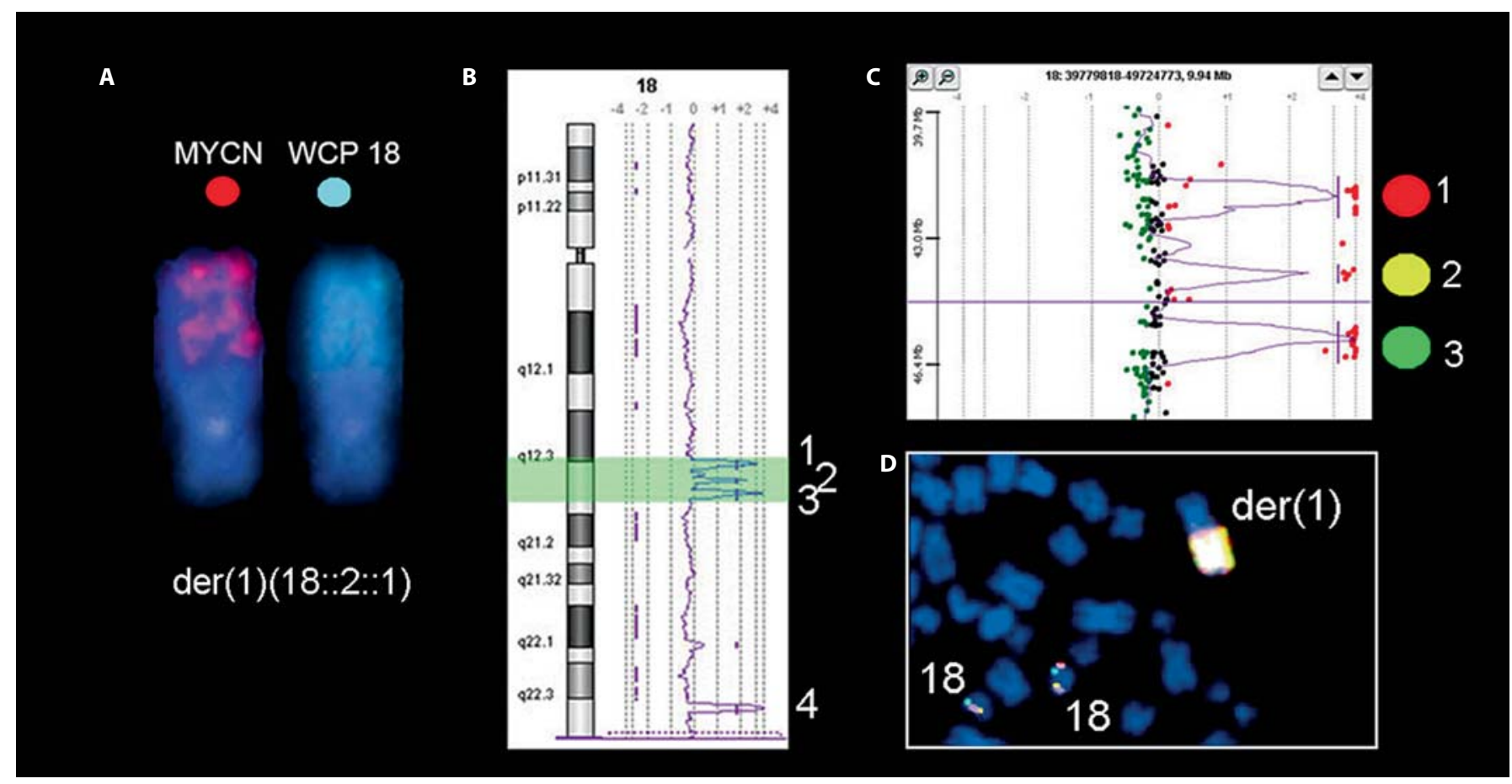

Fig. 2. FISH analysis of the 18q amplicon of Y79. A FISH analysis with a whole-chromosome paint probe for chromosome 18 and a $M Y C N$ probe confirms co-amplification of MYCN and chromosome 18-derived sequences in the hsr on $\operatorname{der}(1)(18:: 2:: 1)$. Shown are the non-merged images of the individual probes on the same der(1) chromosome for better visualization of the co-localization. B aCGH profile for chromosome 18 shows 4 regions of amplification along $18 \mathrm{q}$ at $18 \mathrm{q} 21$ and $18 \mathrm{q} 22$. C aCGH profile showing the presence of 3 amplified regions within the 18q21 locus and the color of FISH probes used to confirm their amplification within the hsr (shown in D). D Locus-specific FISH (shown in C) hybridized to the hsr on der(1)(18::2::1), confirms the co-amplification of the 3 identified loci by aCGH. Two normal chromosomes 18 within the metaphase spread are also shown to contain all 3 probes in single copies.
Using a whole chromosome painting probe for chromosome 18 and locus-specific probe for MYCN (fig. 2A), the presence of material from both chromosomes were confirmed within the der (1), aCGH also identified additional amplification of regions from 18q21.1 and one from $18 \mathrm{q} 23$ in Y79 (fig. 2B), consistent with previous findings [Godbout et al., 1997]. Three color locus-specific FISH using probes from each of the peaks confirmed their presence within the hsr (fig. 2C, D; data from peak 4 not shown), yielding a revised integrated SKY/mBAND karyotype of +der(1)(hsr18q21.1,hsr18q23::hsr2p24 $\rightarrow 2$ p16 $\rightarrow 2$ p12::1p11 $\rightarrow$ 1qter). Together with FISH analysis using the MYCN probe, these data indicate that the NAG/DDX1/ MYCN hsr unit (figs. 1E, 2A) is comprised of an integrated 2p24 and chromosome 18 amplicon in 2 distinct amplification blocks that are separated apparently by only chromosome 18-derived amplicons (fig. 2A). Multi-color FISH with BAC probes targeting 18q21.1 confirmed the structure of this hsr (fig. 2C, D). The junctions of each amplicon from 18q21.1 were in close proximity to CNVs: peak 1 was flanked by CNV 0618, peak 2 was flanked telomerically by CNV 1965, and peak 3 was flanked by CNVs 1966 and 1967. Additionally, the breakpoint at the transition of chromosome $2 \mathrm{p} 16 \rightarrow \mathrm{p} 22$ into the hsr unit shows CNVs 0363 and 1504 within the region of copy-number transition from then net gain, to normal copy.

\section{Integrated Molecular Cytogenetic and Genomic Analysis of GOTO and IMR32: Intrachromosomal Rearrangements of $2 p$ Associated with MYCN Amplification}

SKY analysis showed that the karyotype of GOTO is diploid with relatively simple structural aberrations (online suppl. table 2; online suppl. fig. 2), which involve chromosome 2 (fig. 3A, i-v). One normal chromosome 2 was identified, as was one der(2) with an unbalanced translocation with chromosome 15 , resulting in the deletion of 2p24 (fig. 3A, iii). The GOTO karyotype in- 


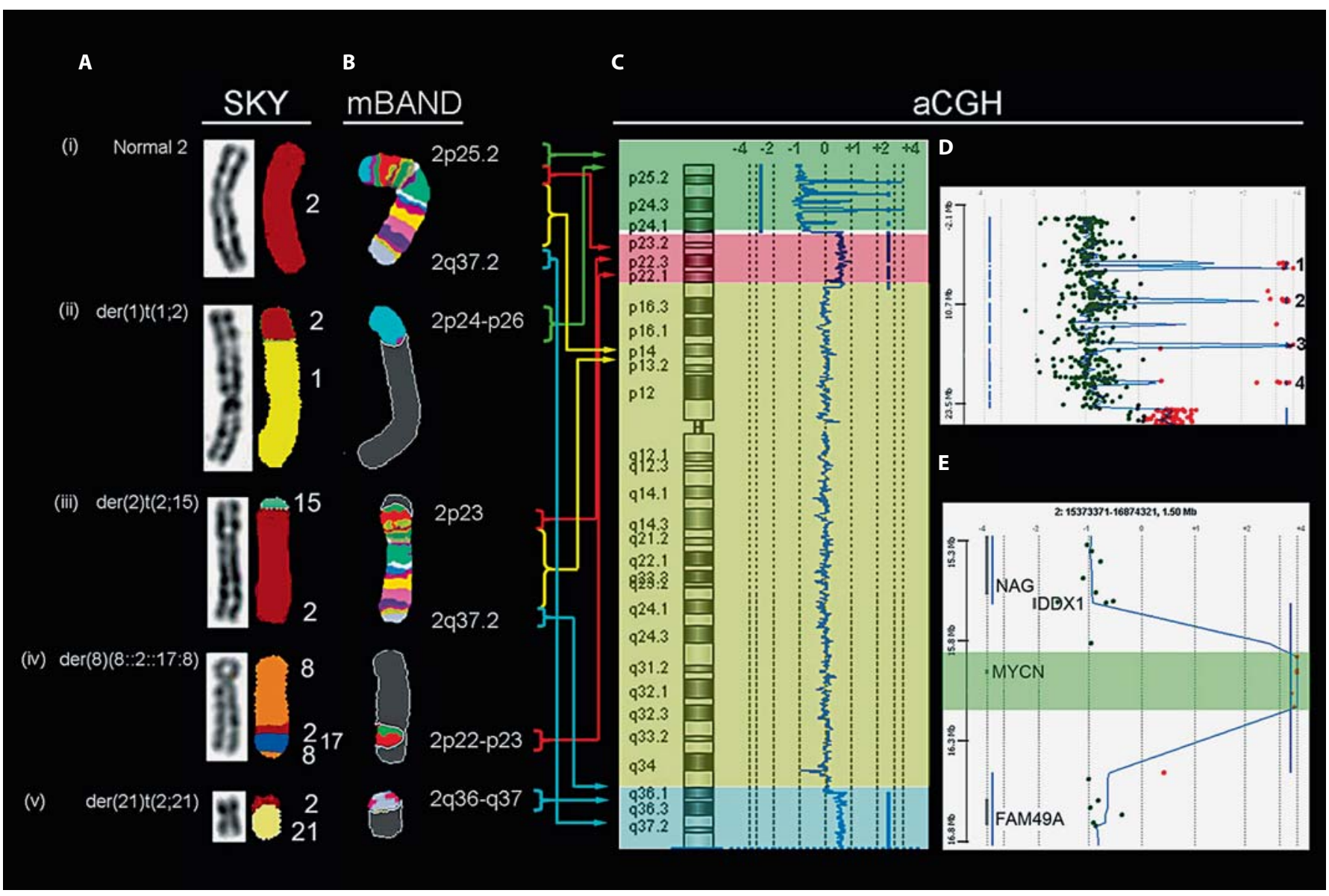

Fig. 3. Integrated molecular cytogenetic and genomic analyses of GOTO. A SKY analysis detects one normal chromosome 2 (i) and 4 additional chromosomes involving sequences from chromosome 2 (ii-v). B Chromosome 2-specific mBAND analysis of (i-v) confirms amplification of 2p24 in the hsr on the der(1) chromosome shown in (ii). C aCGH profile of chromosome 2 reveals a complex pattern of amplifications and intervening deletions in the 2 p $24 \rightarrow 2$ pter region. Chromosomal gain was detected in 2 p23.2 $\rightarrow 2 \mathrm{p} 22.1$ (red arrows) and 2q36.1 $\rightarrow$ 2qter (aqua arrows) with corresponding copy-number changes detected in mBAND. D High-resolution aCGH of 2 p $24 \rightarrow 2$ pter shows regions of gain (red dots) and regions of loss (green dots). Four main peaks of amplification were identified, where at least 2 contiguous features were present. Each peak of amplification was separated by and interspersed with regions of loss. This is consistent with loss of the 2 p24 $\rightarrow$ p25 region detected by SKY (A, iii) and mBAND (B, iii) analysis. E High-resolution aCGH shows that only $M Y C N$ was detected in the 2 p24 amplicon identified in peak 3 . cludes a der(1)t $(1 ; 2)$ chromosome that carries a $M Y C N$ containing hsr (fig. 3A, ii). Sequences from chromosome 2 were also associated with a complex insertion involving chromosomes 8 and 17 (der(8)(8::2::17::8) (fig. 3A, iv) and with an unbalanced translocation involving chromosome 21 (fig. 3A, v). Chromosome 2-specific mBAND analysis confirmed the presence of the normal chromosome 2 (fig. 3B, i) and a deletion of $2 \mathrm{p} 24 \rightarrow \mathrm{p} 25.3$ on the der(2)t(2;15) (fig. 3B, iii). The hsr on der(1) includes sequences from 2 p24 $\rightarrow$ p 25.3 (fig. $3 \mathrm{~B}$, ii), while the complex insertion on der(8), involving chromosomes 17 and
2 , includes sequences from 2 p22 $\rightarrow$ p 23 (fig. 3B, iv) and the unbalanced translocation on der(21) sequences from $2 \mathrm{q} 36 \rightarrow \mathrm{q} 37$.

aCGH analysis of GOTO confirmed the SKY and mBAND findings, indicating a complex pattern of amplification and net deletion in 2 p24 $\rightarrow 25.3$ (fig. 3C, D). This amplicon included 4 evenly spaced amplifications (fig. 3D). A net deletion of one copy was detected in the intervening regions, which is consistent with the presence of one normal copy of chromosome 2 in the karyotype. aCGH data also revealed that only MYCN was am- 
Fig. 4. FISH analysis of the MYCN amplicon in GOTO. Shown are the multicolor locus-specific FISH results for NAG/DDX1, FAM49A and MYCN confirms that only $M Y C N$ is amplified in the hsr on $\operatorname{der}(1) \mathrm{t}(1 ; 2)$, while $N A G / D D X 1$ and FAM49A are present as single-copy genes at 2p24 in normal chromosome 2p24. MYCN, NAG/DDX1 and FAM49A fail to hybridize to der(2)t $(2 ; 15)$. Each image shows the same metaphase spread with only 2 of the locus-specific probes for better visualization in a non-merged image state.

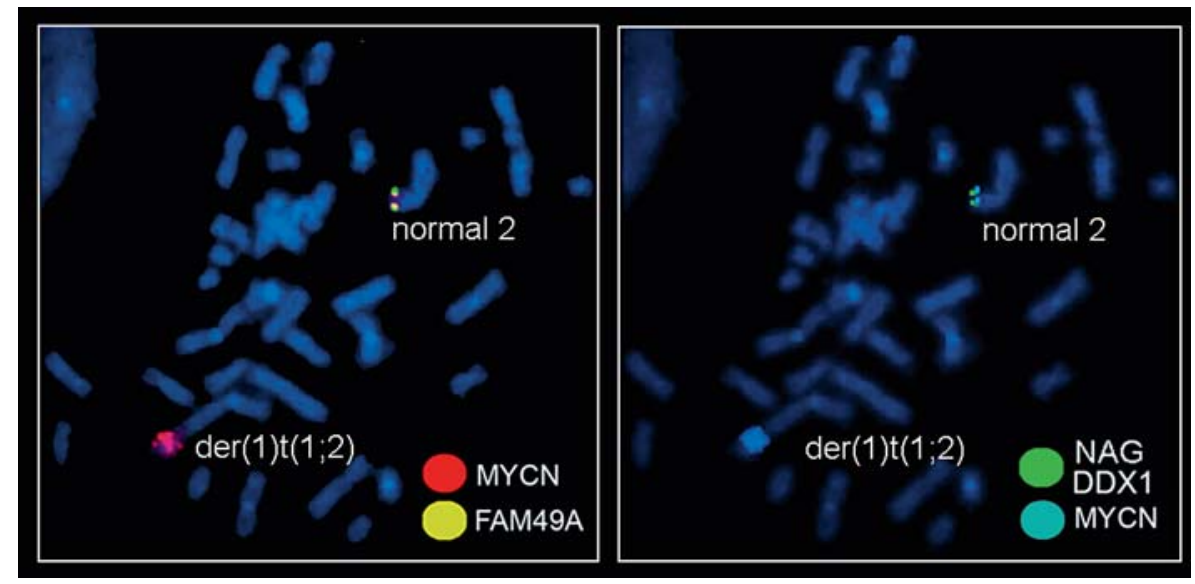

plified in peak 3 (fig. 3D, E). This result was confirmed by multi-color FISH, which failed to show hybridization of BAC probes for FAM49A or DDX1 in this hsr (fig. 4). The normal chromosome 2 had single copies of DDX1, MYCN and FAM49A (fig. 4), while der $(2) \mathrm{t}(2 ; 15)$ had a deletion of these genes. CNV1493, which was also shown to be in the region of copy-number transition for Y79, was also identified near the region of copy-number transition between peak 1 and the start of the 'valley' between peaks 1 and 2; however, no other CNVs appeared to be affected within the regions of copy-number change between the amplified loci of the other regions.

SKY analysis of IMR32 revealed a diploid genome (online suppl. table 2; online suppl. fig. 3) and very few structural aberrations with the exception of a complex rearrangement involving chromosomes 1 and 17. This der(1) (2::17::1) is present in duplicate and carries a $M Y C N-c o n-$ taining hsr (fig. 5A, iii, iv). Because IMR32 also has 2 normal copies of chromosome 2 (fig. 5 , i, ii), the initial amplification was most likely associated with a complex unbalanced translocation involving chromosomes 1, 17 and 2 , followed by duplication of this chromosome. Chromosome 2-specific mBAND confirmed the presence of the 2 normal copies of chromosome 2 as well as amplification of 2 p24 and 2 p14 on the duplicate der(1) containing the hsrs (fig. 5B, i-iv).

aCGH verified the observed diploid status of IMR32, and the gains and losses indicated by SKY analysis (online suppl. fig. 3) including amplification of MYCN and 2 p14, which was previously shown to contain the MEIS1 gene [Jones et al., 2000; Spieker et al., 2001] (fig. 5C). CNV0569 localized in the MYCN amplicon (fig. 5D) was the only $\mathrm{CNV}$ apparently affected by copy-number gains in IMR32. aCGH showed that the amplification unit contains FAM84A to MYCN (fig. 5D). This result was confirmed by multi-color FISH, which showed amplification of FAM84A, MYCN, DDX1, and NAG, but not FAM49A. Therefore, a breakpoint lies between FAM49A and MYCN (fig. 6A-D). The normal copies of chromosome 2 in IMR32 carried no copy-number alterations (i.e. singlecopy genes throughout). This locus-specific FISH analysis also showed that the intensity of the hybridization signal in the hsr varies; whereby inverted DAPI images suggest that the darker region corresponds to 2 p24 and the lighter region corresponds to $2 \mathrm{p} 14$. In summary, the karyotypes of IMR32 and GOTO include intra-chromosomal rearrangements associated with complex amplicons containing variable regions of $2 \mathrm{p}$.

\section{Integrated Molecular Cytogenetic and Genomic}

Analysis of LAN5: Identification of Two Distinct dmin Populations

LAN5 has $2 \mathrm{dmin}$ populations, one of which includes MYCN [Pandita et al., 1997]. SKY analysis of LAN5 revealed a nearly diploid karyotype, containing 2 clones that differed by the presence of a $\operatorname{der}(8) \mathrm{t}(7: 8)$, (online suppl. fig. 4; online suppl. table 2). Dmins were detected and characterized as sequences from chromosome 2, consistent with the mapping of $M Y C N$ at 2p24 (fig. 7A, iii). The size and number of dmins varied from metaphase to metaphase, also consistent with previous findings [Pandita et al., 1997]. Chromosome 2-specific mBAND analysis revealed 2 normal copies of chromosome 2 and confirmed the presence of 2 p24 material in the dmins (fig. 7B, i-iii). aCGH analysis also confirmed amplification of the 2p24 region containing MYCN (fig. 7C) as well as DDX1 


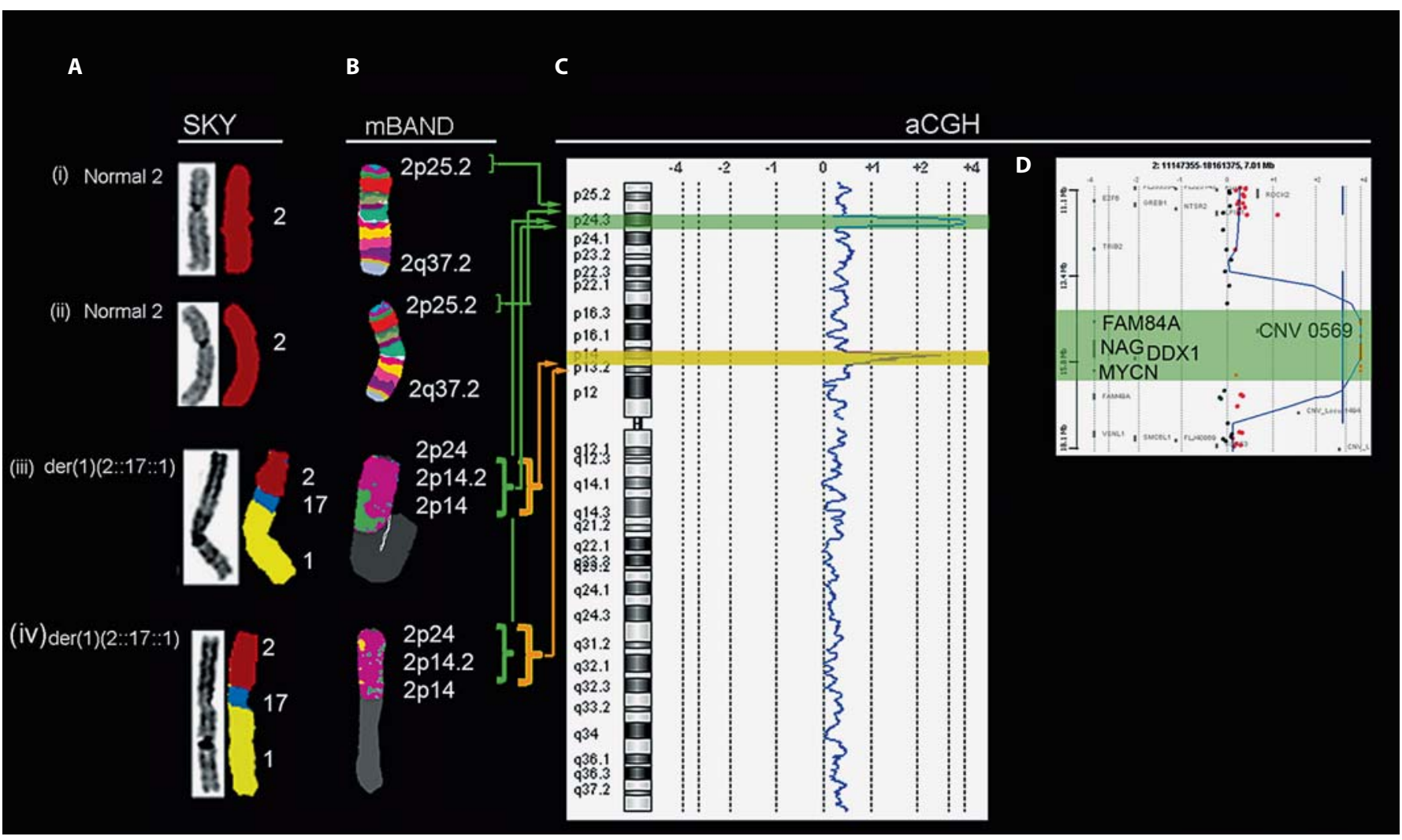

Fig. 5. Integrated molecular cytogenetic and genomic analyses of IMR32. A SKY analysis shows 2 normal copies of chromosome 2 (i, ii) and 2 copies of a der(1)t(2::17::1) (iii, iv). B Chromosome 2-specific mBAND analysis shows that the $\operatorname{der}(1) t(2:: 17:: 1)$ lacks gross intra-chromosomal rearrangements of the chromosome 2 sequences but has an hsr with amplification of sequences from 2p14.2 and 2p24. C aCGH for chromosome 2 confirms amplification of 2 p24 and 2p14.2. D High-resolution aCGH of the 2p24 region confirms co-amplification of $M Y C N, D D X 1, N A G$ and FAM84A. and FAM49A (fig. 7D), while FAM84A and VSNL1 were present at the level of 2 copies (fig. 7D). Interestingly, CNV 0569 and CNV 1494 were also detected in the transition regions flanking the higher copy-number region. Multicolor FISH using BAC clones confirmed the aCGH results (fig. 8A) showing that the dmin contained only MYCN and DDX1. The normal copies of chromosome 2 (arrows) hybridized to FISH probes for FAM84A, DDX1, MYCN, and VSNL1. aCGH and SKY data were in agreement with each other, with the exception that aCGH showed amplification of sequences from 6p12.3 (chr6:49,506,950$50,848,705)$, which SKY failed to detect. This region was included in a BAC clone (RP11-624I21) that contained sequences from CRISP2 and PGK2 (fig. 8B). FISH analysis using this BAC revealed small insertions of sequences from $6 \mathrm{p} 12.3$ on other chromosomes as indicated by asterisks in figure $8 \mathrm{C}$ (left) as well as a distinct population of dmins that do not include 2p24 (i.e. no MYCN) co-ampli- fied within the $6 \mathrm{p} 12.3 \mathrm{dmins}$ (fig. $8 \mathrm{C}$, right). The amplicon from 6p12.3 included CRISP2 and PGK2 and was associated with CNV0145 and 0579 in the copy-number transition regions.

\section{Discussion}

This study presents a comprehensive molecular cytogenetic and genomic analysis of MYCN amplicons in 4 human neuronal cell lines, Y79, GOTO, IMR32, and LAN5. The integrative use of these diverse molecular cytogenetic techniques clearly exemplifies the limitations of using just one technique alone [Bayani and Squire, 2007]. Although the karyotypes of these cell lines are nearly diploid, all four cell lines carried one or more complex chromosomal aberrations associated with MYCN amplification. The data from the analyses of these cell lines reveal 


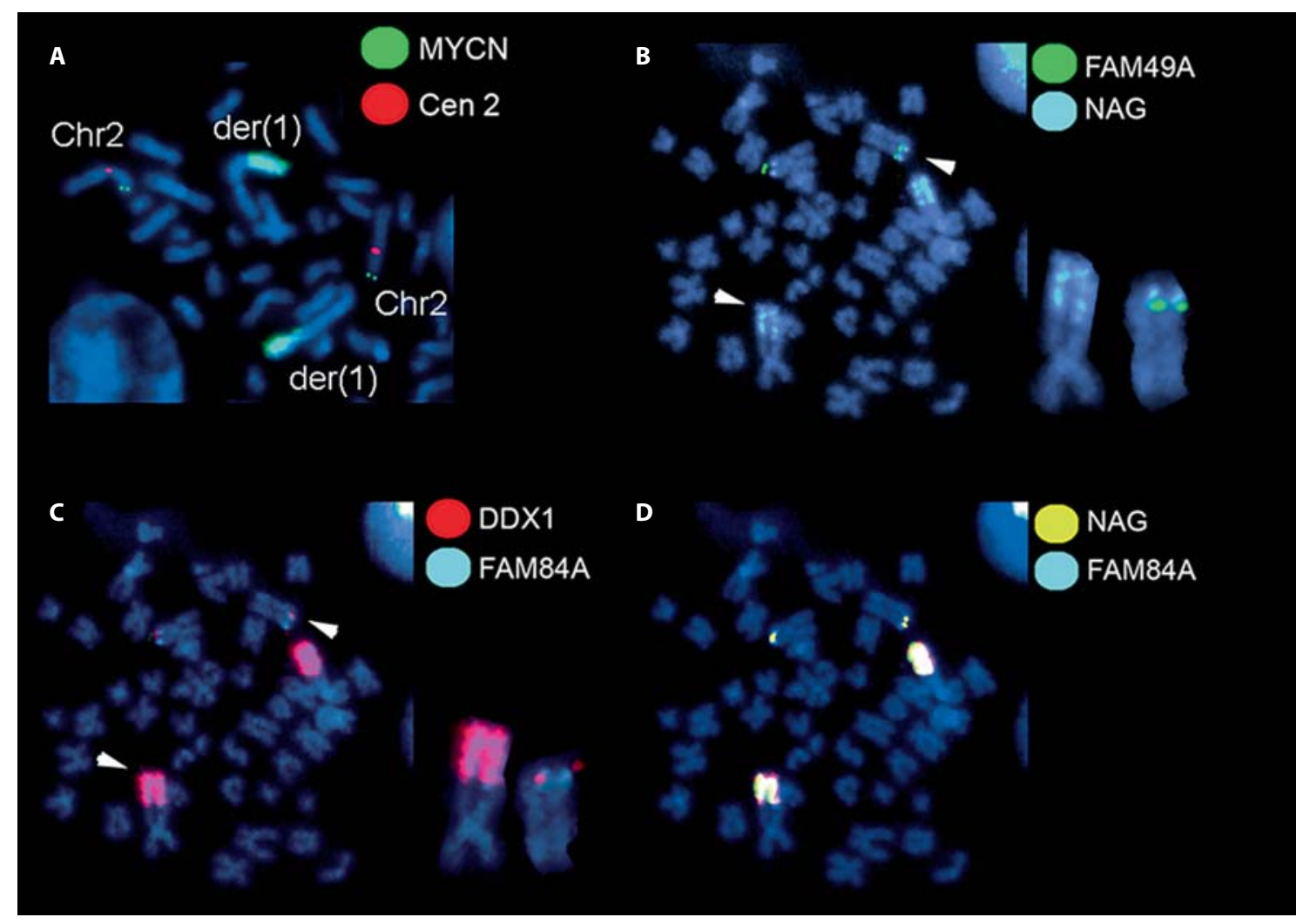

Fig. 6. FISH analysis of the MYCN amplicon in the neuroblastoma cell line IMR32. Multi-color locus-specific FISH for MYCN (A), FAM49A (B), NAG (B and C), DDX1 (C) and FAM84A (C, D) confirmed co-amplification of MYCN, DDX1, NAG, and FAM84A on the der(1) chromosome in IMR32. B-D Images show the same metaphase spread with 2 of the locus-specific probes for better visualization in the non-merged image state.

complex structural rearrangements and losses involving the MYCN locus and other regions of the genome, indicating multiple intricate rearrangements were associated with each amplicon. The results provided evidence for the following cell-line specific chromosomal changes: in Y79 cells, a hsr that includes co-amplification of MYCN and 4 segments of chromosome 18q; in GOTO, an hsr that includes co-amplification of 4 evenly-spaced chromosome segments between 2p24.1 and 2p25.1; in IMR32, an hsr distal to MYCN that includes co-amplification of multiple regions of $2 \mathrm{p}$; in LAN5, 2 sets of dmins, one involving a MYCN amplicon and one involving an amplicon from chromosome $6 \mathrm{p} 12$. CNV sequences were detected in close proximity to copy-number transition regions immediately adjacent to both hsr- and dmin-associated amplicons.

There are several mechanisms that have been proposed to explain the formation of $\mathrm{dmin} / \mathrm{hsr}$ and gene amplification [Windle and Wahl, 1992; Schwab, 2004;
Albertson, 2006]. One of the earliest and most coherent models is the breakage-fusion-bridge (BFB) mechanism [McClintock, 1942], in which a chromosome break is followed by end-to-end fusion after DNA repair, which generates a mitotically unstable dicentric chromosome. A hallmark of BFB-based amplification is the presence of amplicons at the resident location of the gene. However, in our current analyses, it was rare to observe the presence of the MYCN amplicon at 2p24. This would suggest that other alternate mechanism(s) such as fork stalling and template switching, and microhomology-mediated breakinduced replication [Hastings et al., 2009] may be involved in high copy number amplification of the MYCN gene.

Within this study group, Y79 and GOTO exhibited loss of sequences from the $M Y C N$ resident site, whereas LAN5 and IMR32 retained the MYCN gene at the resident site. Bignell et al. [2007] postulated that tumors with structural alterations (deletion and/or rearrangements) at the resident site are more compatible with models in 


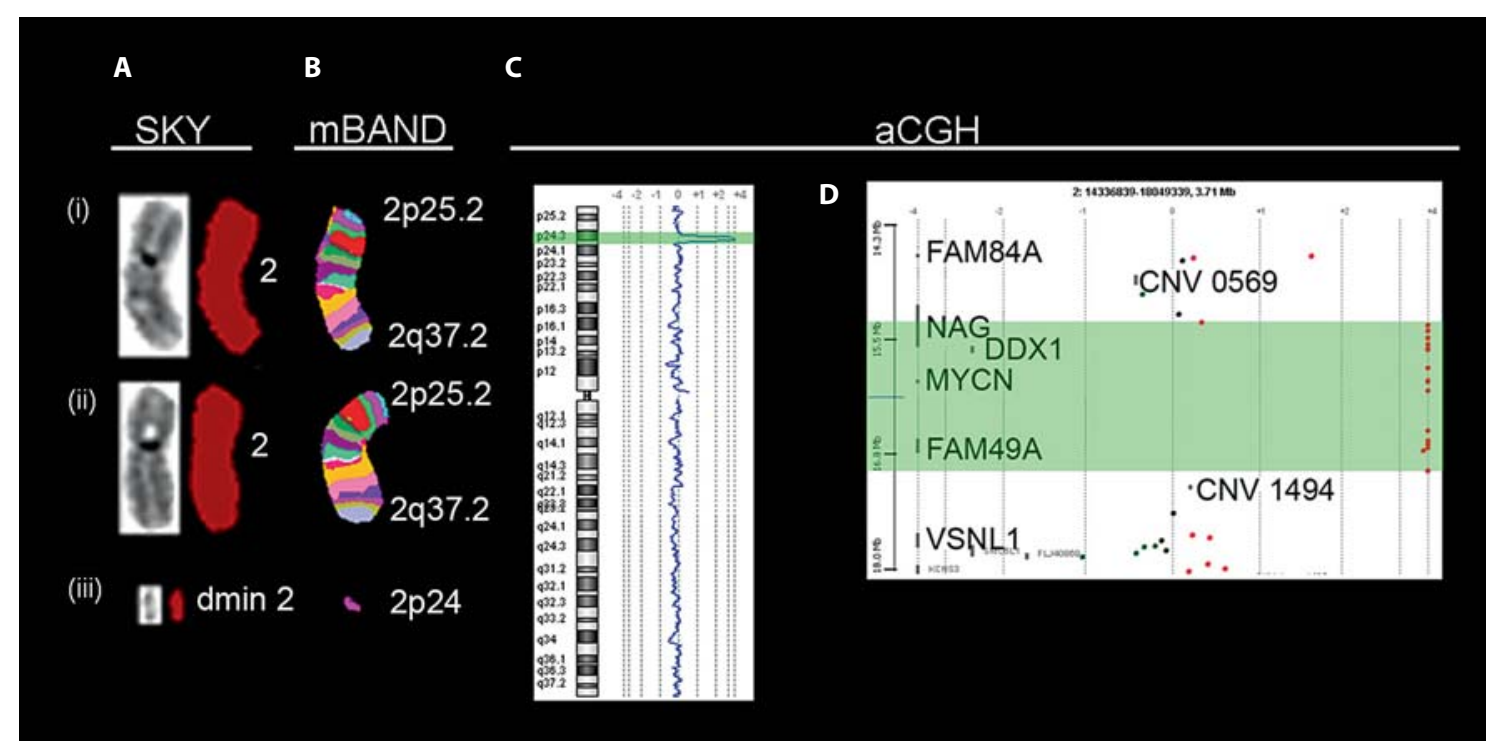

Fig. 7. Integrated molecular cytogenetic and genomic analyses of the neuroblastoma cell line LAN5. A SKY analysis shows 2 normal copies of chromosome 2 (i, ii) and extra-chromosomal dmins carrying sequences from chromosome 2 (iii). B Chromosome 2-specfic mBAND detects no gross intra-chromosomal rearrangement of chromosome 2 (i, ii), and confirms 2p24 sequences in the dmins (iii). C, D aCGH for chromosome 2 shows copynumber increases for NAG to FAM49A in the 2p24 region. A transition copy-number region includes CNV0569 and CNV1494.

which there is a looping out and excision of DNA to form double minute chromosomes. The loss of MYCN in one copy of chromosome 2 in Y79 and GOTO would be consistent with this mechanism. However, the lack of dmins in both cell lines would require that the dmins reintegrate back into the genome as hsrs. Another mechanism that may be helpful in considering the amplification process in these cell lines is based on the idea that simultaneous fragmentation of multiple chromosomal regions leads to amplification [Gibaud et al., 2010]. Such fragmentation has been shown to be induced by replication stress, resulting in chromosomal rearrangements and dmin formation [Stevens et al., 2007]. Further studies involving sequencing of the 2 p24 region and amplicon structures in these cell lines would provide more insight into the precise mechanisms involved.

We have previously reported a co-amplification of MYCN and the ATP synthase alpha-subunit gene (ATP$5 A$ ) from chromosome 18 in the Y79 hsr [Godbout et al., 1997]. Here, we show that 4 distinct regions of chromosome 18 are co-amplified with MYCN in the hsr in Y79. The mechanism by which this complex hsr arose is unknown. However, we speculate that complex chromosomal rearrangements might play a role in generating hsrs with such complex patterns of amplification. For example, a 3-way translocation involving chromosomes 1, 2 and 18 may have generated an intermediate leading to the Y79 hsr, reminiscent of findings by Mitsui et al. [2007] based on the co-amplification of MYCC, ERBB2 and EGFR in patient samples. Our FISH analysis of the $\mathrm{Y} 79 \mathrm{hsr}$ showed that FAM49A and FAM84A from cytoband 2p24 localize to the hsr junction at 2 p16 (fig. $1 \mathrm{E}$, right), but are not detected in the hsr itself. It is tempting to speculate that these 2 genes are remnants of a precursor amplicon, possibly a dmin, that integrated into 2p16 and that the Y79 hsr may have formed by unequal sister-chromatid exchange facilitated by CNVs in the amplicon precursor (see below).

The presence of 4 relatively evenly spaced regions of low and high copy-number alteration (fig. 3C, D), observed in GOTO, may possibly be a result of multiple amplification cycles involving structural damage to the chromosomal region. The hsr in IMR32 is a complex amplification involving MYCN from 2p24 and MEIS1 from 2p14 [Jones et al., 2000; Spieker et al., 2001]. FAM84A and CNV0569 are included in the 2p24 amplicon. However, there are no CNVs close to MEIS1, indicating that CNVs may not have been involved in generating the IMR32 hsr. Several groups have suggested that the driver in this amplicon is MEIS1 [Jones et al., 2000; Spieker et al., 2001; Geerts et al., 2003]. 

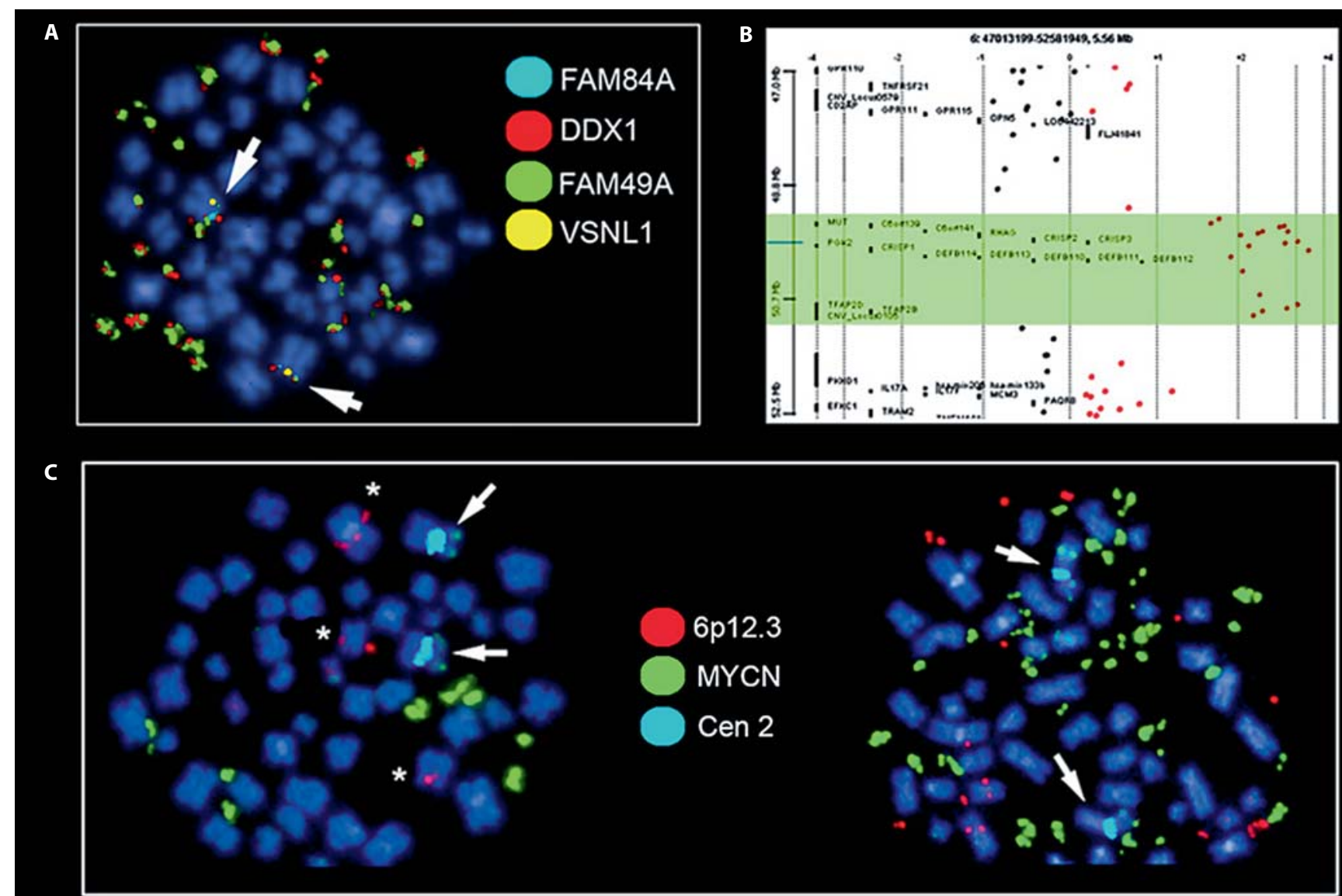

Fig. 8. FISH analysis of the MYCN amplicon on dmins in LAN5. A Multi-color, locus-specific FISH using BAC clones confirms amplification from NAG to FAM49A in the chromosome 2-derived dmins and single-copy genes in the 2p24 locus on the normal chromosome 2 (arrow). B aCGH shows amplification of 6p12.3. C The multi-color locus-specific FISH reveals amplifica- tion of $6 \mathrm{p} 12.3$ sequences on dmins (right) or integrated in various chromosomal locations (left). Dmins carried the amplicon from $6 \mathrm{p} 12.3$ or the $M Y C N$ amplicon, but not both (right). Chromosome 2 is indicated by arrows and while the integration of the $6 \mathrm{p} 12.3$ locus into chromosomes is indicated by the asterisk $\left(^{*}\right)$.
LAN5 has 2 distinct populations of dmins: one dmin has a focal amplicon centered on $M Y C N$ that includes NAG, DDX1 and FAM49A, and the second dmin includes CRISP2 and PGK2 from 6p12.3. Both amplicons are flanked by CNVs, which may have facilitated acquisition of dmins in this cell line.

$\mathrm{CNV}$ sequences were detected throughout the regions of 2 p24 affected by gene amplification in all 4 cell lines analyzed in this study (http://projects.tcag.ca/variation/). CNVs are intrinsically unstable [Zhang et al., 2009] and are often associated with chromosomal alterations in cancer cells [Hastings et al., 2009]. CNVs may increase the rate of DNA replication errors, by causing fork stalling and template switching [Lee et al., 2007]. CNVs con- tain tracts of microhomology that could lead to higher rates of unequal sister-chromatid exchange in precursor amplicons. Interestingly, it was recently reported that inherited CNVs at 1q21.1 are associated with a higher risk of developing neuroblastoma [Diskin et al., 2009]. Thus, it is possible that $\mathrm{CNV}$ sequence clusters in the general vicinity of MYCN may have promoted genetic instability and thereby facilitated chromosome rearrangements leading to high copy MYCN amplification.

Several recent studies also suggest that neuroblastoma patients with complex MYCN amplicons have more severe symptoms than patients with simple $M Y C N$ amplicons [Mosse et al., 2007; Caren et al., 2008; Fix et al., 2008]. Fix et al. [2008] showed that sequences that flank 
amplified regions are deleted in approximately $20 \%$ of MYCN amplicons; in addition to observations by Corvi et al. [1994] showing that a single copy of $M Y C N$ was retained at 2p24.3 in cell lines with MYCN amplification, we observed that endogenous $M Y C N$ at 2p24 was deleted in Y79 and GOTO.

In summary, the results presented here provide a comprehensive analysis of the structure and complexity of high copy-number regions associated with MYCN amplification in neuronal cell lines. Additional studies are needed to determine whether patient tumors that exhib- it similar high levels of genomic complexity associated with MYCN amplification represent a distinct molecular subset of neuronal tumors that may require different management approaches.

\section{Acknowledgements}

This work was supported by the Canadian Cancer Society (CCS) and the James Birrell Fund.

\section{References}

Akiyama K, Kanda N, Yamada M, Kato M, Tadokoro K, et al: Structural organization of MYCN amplicons of neuroblastoma tumors, xenografts, and cell lines characterized by the sequences encompassing the $M Y C N$ amplicons in a human neuroblastoma cell line. Genes Chromosomes Cancer 8:15-21 (1993).

-Albertson DG: Gene amplification in cancer. Trends Genet 22:447-455 (2006)

Albertson DG, Collins C, McCormick F, Gray JW: Chromosome aberrations in solid tumors. Nat Genet 34:369-376 (2003).

Bayani J, Squire J: Multi-color FISH techniques. Curr Protoc Cell Biol Chapter 22:Unit 22.5 (2004a).

Bayani J, Squire JA: Preparation of cytogenetic specimens from tissue samples. Curr Protoc Cell Biol Chapter 22:Unit 22.2 (2004b)

Bayani J, Squire JA: Fluorescence in situ hybridization (FISH). Curr Protoc Cell Biol Chapter 22:Unit 22.4 (2004c).

Bayani J, Squire JA: Application and interpretation of FISH in biomarker studies. Cancer Lett 249:97-109 (2007).

-Bignell GR, Santarius T, Pole JC, Butler AP, Perry J, et al: Architectures of somatic genomic rearrangement in human cancer amplicons at sequence-level resolution. Genome Res 17: 1296-1303 (2007).

Brodeur GM, Seeger RC, Schwab M, Varmus HE, Bishop JM: Amplification of N-MYC in untreated human neuroblastomas correlates with advanced disease stage. Science 224: 1121-1124 (1984)

- Caren H, Erichsen J, Olsson L, Enerback C, Sjoberg RM, et al: High-resolution array copy number analyses for detection of deletion, gain, amplification and copy-neutral $\mathrm{LOH}$ in primary neuroblastoma tumors: four cases of homozygous deletions of the CDKN2A gene. BMC Genomics 9:353 (2008).

Corvi R, Amler LC, Savelyeva L, Gehring M, Schwab M: $M Y C N$ is retained in single copy at chromosome 2 band p23-24 during amplification in human neuroblastoma cells. Proc Natl Acad Sci USA 91:5523-5527 (1994).
Diskin SJ, Hou C, Glessner JT, Attiyeh EF, Laudenslager $M$, et al: Copy number variation at 1q21.1 associated with neuroblastoma. Nature 459:987-991 (2009).

Fix A, Lucchesi C, Ribeiro A, Lequin D, Pierron $\mathrm{G}$, et al: Characterization of amplicons in neuroblastoma: high-resolution mapping using DNA microarrays, relationship with outcome, and identification of overexpressed genes. Genes Chromosomes Cancer 47:819-834 (2008).

Geerts D, Schilderink N, Jorritsma G, Versteeg $\mathrm{R}$ : The role of the MEIS homeobox genes in neuroblastoma. Cancer Lett 197:87-92 (2003).

Gibaud A, Vogt N, Hadj-Hamou NS, Meyniel JP, Hupe P, et al: Extrachromosomal amplification mechanisms in a glioma with amplified sequences from multiple chromosome loci. Hum Mol Genet 19:1276-1285 (2010).

Godbout R, Squire J: Amplification of a dead box protein gene in retinoblastoma cell lines. Proc Natl Acad Sci USA 90:7578-7582 (1993).

Godbout R, Pandita A, Beatty B, Bie W, Squire JA: Comparative genomic hybridization analysis of Y79 and FISH mapping indicate the amplified human mitochondrial ATP synthase alpha-subunit gene (ATP5A) maps to chromosome $18 \mathrm{q} 12 \rightarrow \mathrm{q} 21$. Cytogenet Cell Genet 77:253-256 (1997).

Hastings PJ, Lupski JR, Rosenberg SM, Ira G: Mechanisms of change in gene copy number. Nat Rev Genet 10:551-564 (2009).

Jones TA, Flomen RH, Senger G, Nizetic D, Sheer $\mathrm{D}$ : The homeobox gene MEIS1 is amplified in IMR-32 and highly expressed in other neuroblastoma cell lines. Eur J Cancer 36:23682374 (2000).

Kitajima K, Haque M, Nakamura H, Hirano T, Utiyama H: Loss of irreversibility of granulocytic differentiation induced by dimethyl sulfoxide in HL-60 sublines with a homogeneously staining region. Biochem Biophys Res Commun 288:1182-1187 (2001).
Kohl NE, Kanda N, Schreck RR, Bruns G, Latt SA, et al: Transposition and amplification of oncogene-related sequences in human neuroblastomas. Cell 35:359-367 (1983).

Lee JA, Carvalho CM, Lupski JR: A DNA replication mechanism for generating nonrecurrent rearrangements associated with genomic disorders. Cell 131:1235-1247 (2007).

Lim G, Karaskova J, Vukovic B, Bayani J, Beheshti B, et al: Combined spectral karyotyping, multicolor banding, and microarray comparative genomic hybridization analysis provides a detailed characterization of complex structural chromosomal rearrangements associated with gene amplification in the osteosarcoma cell line MG-63. Cancer Genet Cytogenet 153:158-164 (2004).

-Lipson D, Aumann Y, Ben-Dor A, Linial N, Yakhini Z: Efficient calculation of interval scores for DNA copy number data analysis. J Comput Biol 13:215-228 (2006).

McClintock B: The fusion of broken ends of chromosomes following nuclear fusion. Proc Natl Acad Sci USA 28:458-463 (1942).

Mitsui F, Dobashi Y, Imoto I, Inazawa J, Kono K, et al: Non-incidental coamplification of $M Y C$ and $E R B B 2$, and $M Y C$ and EGFR, in gastric adenocarcinomas. Mod Pathol 20: 622-631 (2007).

Moreau LA, McGrady P, London WB, Shimada $\mathrm{H}, \mathrm{Cohn} \mathrm{SL}$, et al: Does MYCN amplification manifested as homogeneously staining regions at diagnosis predict a worse outcome in children with neuroblastoma? A children's oncology group study. Clin Cancer Res 12: 5693-5697 (2006).

-Mosse YP, Diskin SJ, Wasserman N, Rinaldi K, Attiyeh EF, et al: Neuroblastomas have distinct genomic DNA profiles that predict clinical phenotype and regional gene expression. Genes Chromosomes Cancer 46:936949 (2007).

Narayanan V, Mieczkowski PA, Kim HM, Petes TD, Lobachev KS: The pattern of gene amplification is determined by the chromosomal location of hairpin-capped breaks. Cell 125: 1283-1296 (2006). 
Noguchi T, Akiyama K, Yokoyama M, Kanda N, Matsunaga T, Nishi Y: Amplification of a dead box gene (DDX1) with the MYCN gene in neuroblastomas as a result of cosegregation of sequences flanking the MYCN locus. Genes Chromosomes Cancer 15:129-133 (1996).

-Paderova J, Orlic-Milacic M, Yoshimoto M, da Cunha Santos G, Gallie B, Squire JA: Novel $6 p$ rearrangements and recurrent translocation breakpoints in retinoblastoma cell lines identified by spectral karyotyping and mBAND analyses. Cancer Genet Cytogenet 179:102-111 (2007).

Pandita A, Godbout R, Zielenska M, Thorner P, Bayani J, Squire JA: Relational mapping of $M Y C N$ and DDX1 in band 2p24 and analysis of amplicon arrays in double minute chromosomes and homogeneously staining regions by use of free chromatin FISH. Genes Chromosomes Cancer 20:243-252 (1997).

Prasad M, Bernardini M, Tsalenko A, Marrano P, Paderova J, et al: High definition cytogenetics and oligonucleotide acgh analyses of cisplatin-resistant ovarian cancer cells. Genes Chromosomes Cancer 47:427-436 (2008).
Schwab M: MYCN amplifications in neuroblastoma, in Brodeur G, Sawada T, Tsuchida Y, Voute P (eds): Neuroblastomas, pp 75-84 (Elsevier, Amsterdam 2000).

Schwab M: MYCN in neuronal tumours. Cancer Lett 204:179-187 (2004).

Schwab M, Amler LC: Amplification of cellular oncogenes: a predictor of clinical outcome in human cancer. Genes Chromosomes Cancer 1:181-193 (1990).

Schwab M, Alitalo K, Klempnauer KH, Varmus HE, Bishop JM, et al: Amplified DNA with limited homology to MYC cellular oncogene is shared by human neuroblastoma cell lines and a neuroblastoma tumour. Nature 305: 245-248 (1983).

Schwab M, Ellison J, Busch M, Rosenau W, Varmus HE, Bishop JM: Enhanced expression of the human gene N-MYC consequent to amplification of DNA may contribute to malignant progression of neuroblastoma. Proc Natl Acad Sci USA 81:4940-4944 (1984).
Shimizu N, Ochi T, Itonaga K: Replication timing of amplified genetic regions relates to intranuclear localization but not to genetic activity or G/R band. Exp Cell Res 268:201-210 (2001).

-Spieker N, van Sluis P, Beitsma M, Boon K, van Schaik BD, et al: The MEIS1 oncogene is highly expressed in neuroblastoma and amplified in cell line IMR32. Genomics 71:214221 (2001).

-Squire J, Goddard AD, Canton M, Becker A, Phillips RA, Gallie BL: Tumour induction by the retinoblastoma mutation is independent of $\mathrm{N}-\mathrm{MYC}$ expression. Nature 322:555-557 (1986).

-Stevens JB, Liu G, Bremer SW, Ye KJ, Xu W, et al: Mitotic cell death by chromosome fragmentation. Cancer Res 67:7686-7694 (2007).

-Windle BE, Wahl GM: Molecular dissection of mammalian gene amplification: new mechanistic insights revealed by analyses of very early events. Mutat Res 276:199-224 (1992).

-Zhang F, Gu W, Hurles ME, Lupski JR: Copy number variation in human health, disease, and evolution. Annu Rev Genomics Hum Genet 10:451-481 (2009). 